\title{
CXCL2 as a Prognostic Marker in Breast Cancer is Associated with Immune Infiltration and Regulated by miR-215
}

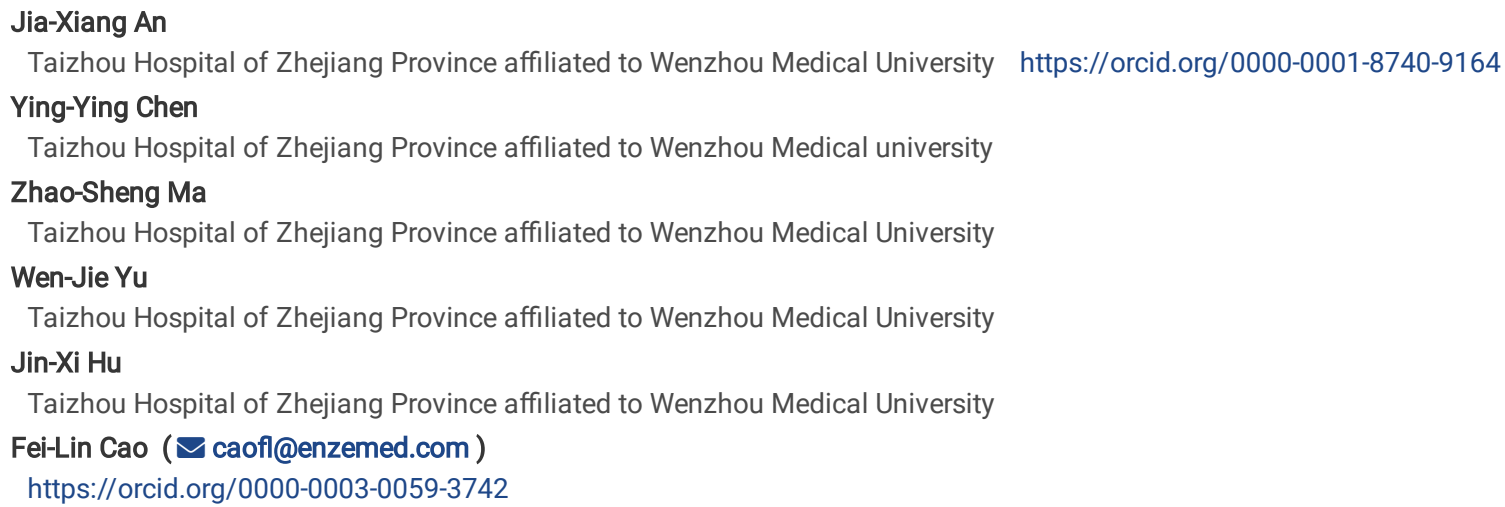

\section{Primary research}

Keywords: CXCL2, breast cancer, immune infiltration, prognosis, miR-532-5p

Posted Date: July 14th, 2020

DOI: https://doi.org/10.21203/rs.3.rs-41001/v1

License: (c) (1) This work is licensed under a Creative Commons Attribution 4.0 International License. Read Full License 


\section{Abstract}

Background: CXCL2 is a part of chemokine superfamily, which encodes secretory proteins involved in immune regulation and inflammation. The correlation between CXCL2 and prognosis of different cancers, tumor infiltrating lymphocytes are not clear.

Methods: We analyzed the expression of CXCL2 and its effect on clinical prognosis through Oncomine database, Tumor Immune Estimation Resource (TIMER) website, Kaplan-Meier plotter, PrognoScan database and Gene Expression Profiling Interactive Analysis (GEPIA). TIMER and GEPIA were used to analyze the correlation between CXCL2 and the gene marker of immune infiltration. StarBase was used to predict the miRNA that may regulate CXCL2. The relationship between miR-532-5p and CXCL2 was detected by qRT-PCR. Kaplan-Meier plotter was used to evaluate the impact of miR-532-5p on clinical prognosis.

Results: PrognoScan, Kaplan-Meier plotter and GEPIA database analysis showed that low expression of CXCL2 was associated with poor disease-specific survival time (DSS), relapse-free survival time (RFS) and overall disease survival (OS) in breast cancer patients. In addition, low expression of CXCL2 was associated with poor OS and RFS in patients with lymph node positive breast cancer. CXCL2 expression was positively correlated with the infiltration of B cells, CD4+T and CD8+T cells, neutrophils and dendritic cells (DCs) in BRCA, mainly in Luminal breast cancer. MiR-532-5p can directly regulate CXCL2 expression. High miR-532-5p expression is significantly correlated with HER2 negative, grade 2 and 3 and poor OS in patients with HER+ER- breast cancer.

Conclusion: CXCL2 is closely related to the prognosis and immune infiltration level of breast cancer patients, it can be regulated by miR-532-5p.

\section{Background}

Breast cancer (BC) is a common malignant tumor worldwide, and its incidence ranks first among female malignant tumors in the world. ${ }^{1,2}$ Invasion and metastasis are the main causes of death in BC patients. ${ }^{3}$ Immune escape mechanism plays an important role in the occurrence and development of malignant tumors. ${ }^{4-6}$ Immunotherapy can improve the immune microenvironment of tumor, make the immune system play the role of anti-cancer again, and achieve the purpose of controlling and even eliminating tumor cells. ${ }^{7}$ However, BC is considered as a "poorly immunogenic" tumor, and its immunotherapy effect is not good. In recent years, the study of immunotherapy for BC has gradually increased, and it has been found that immunotherapy has good effect in some BCs. ${ }^{8}$ Tumor infiltrating lymphocytes (TILs) are the first immune cells found around the cells, which affect the prognosis and curative effect of chemotherapy and immunotherapy, and are of great significance to judge the prognosis of BC patients. ${ }^{9}$ The development of immunotherapy has brought new hope for the treatment of BC. It is urgent to clarify the immunophenotype of breast cancer immune interactions and determine new immunotherapy targets.

CXC-motif chemokine ligand 2 (CXCL2) is a major chemokine of neutrophils. ${ }^{10}$ Its gene is located in $4 \mathrm{q} 21$ of human genome. Production of CXCL2 is associated with macrophages, endothelial cells, epithelial cells and tumor cells. ${ }^{11,12}$ It is involved in neutrophil chemotaxis, inflammation, damage repair, cytoskeleton reconstruction, tumor cell migration, immune response and other important physiological functions by binding with specific receptor $\mathrm{CXCR} 2{ }^{13}-17$ It has been found that CXCL2 secreted from omental adipocytes activates AKT phosphorylation of gastric cancer cells, which directly promotes gastric cancer growth and motility. Consequently, gastric cancer cells were transformed to a more aggressive phenotype, which induces peritoneal metastasis thorough recruitment to the omentum itself. ${ }^{18}$ Another study found that on hepatocellular carcinoma cell, miR-532-5p was negatively correlated with CXCL2 expression. Overexpression of miR-532-5p targeted on CXCL2 decreased cell proliferation, migration and invasion. ${ }^{19}$ In ovarian cancer and endometrial cancer, calcitriol and progesterone can cause the low expression of CXCL2 protein, which can reduce the invasiveness of cancer cells and inhibit the expression of metastasis related proteins. ${ }^{20}$ These findings indicate that CXCL2 plays an important role in the invasion and metastasis of cancer.

Some studies have shown that CXCL2 has the effect of chemokine, which can attract myeloid-derived suppressor cells (MDSC) into the tumor cells in mouse ovarian cancer cells, while MDSC can inhibit the proliferation of CD8 + T cells. ${ }^{21}$ Another study found that CXCL2, produced by M2 macrophages, ${ }^{22}$ was suppressed in PD-L1 KO ID8-inoculated mice. This mechanism may support tumor progression being suppressed as a result of an altered macrophage subset from M2 macrophages producing CXCL1 and CXCL2 to M1 macrophages. ${ }^{23}$ Spleen T lymphocytes of tumor-bearing mice were not only produced by DA-3 breast tumor cells, but also secreted CXCL2. ${ }^{24}$ These findings indicate that CXCL2 has multiple functions in tumor infiltrating lymphocytes. However, the potential function and mechanism of CXCL2 in tumor progression and tumor immunology are still unclear.

In this study, we analyzed the expression of CXCL2 and its correlation with the prognosis of cancer patients through Oncomine, PrognoScan, GEPIA and Kaplan-Meier plotter. At the same time, we also discussed the correlation between CXCL2 and tumor infiltrating immune cells in different tumor microenvironment through Tumor Immune Estimation Resource (TIMER). The results of this study elucidate the important role of CXCL2 in BC, and provide the potential relationship and mechanism between CXCL2 and tumor immune interaction.

\section{Material And Methods Cell culture and transfection}

Human BC cell lines MDA-MB-231 was purchased from American Type Culture Collection. MDA-MB-231 cells were cultured in DMEM medium supplemented with $10 \%$ fetal bovine serum (Gibco; Thermo Fisher Scientific, Inc.) and were maintained at $37^{\circ} \mathrm{C}$ in $5 \% \mathrm{CO} 2$.

miR-negative control (NC) mimics, miR-532-5p mimics, miR-NC inhibitor and miR-532-5p inhibitor were synthesized by GenePharma, Ltd. miR-NC inhibitor and miR-NC mimics served as negative controls. MDA-MB-231 cells was transfected with miR-532-5p mimic, miR-532-5p inhibitor, miR-NC mimic or miR-NC 
inhibitor by Lipofectamine 3000 (Invitrogen; Thermo Fisher Scientific, Inc.) according to the manufacturer's protocol. The following sequences were used: miRNC inhibitor, 5'-UUC UCC GAA CGU GUC ACGU-3'; miR-532-5p inhibitor, 5'-ACG GUC CUA CAC UCA AGG CAU G-3'; miR-NC mimic, 5'-AUU GGA ACG AUA CAG AGA AGA - 3'; and miR-532-5p mimic, 5'-CAU GCC UUG AGU GUA GGA CCG U-3'.

\section{Quantitative real-time PCR of CXCL2}

CXCL2 cDNA was reverse-transcribed according to the manufacturer's instructions (Takara, Dalian, China). GAPDH was used as the reference gene. Quantitative real-time PCR was carried out with SYBR Green (Solarbio, China) in a total volume of $20 \mu \mathrm{L}$ using an Exicycler 96 Real-Time Quantitative Thermal Block (Bioneer, Daejeon, Korea). The reactions were incubated at $94^{\circ} \mathrm{C}$ for $10 \mathrm{~min}$; followed by 40 cycles of $94^{\circ} \mathrm{C}$ for $10 \mathrm{~s}, 60^{\circ} \mathrm{Cfor} 20 \mathrm{~s}$, and $72{ }^{\circ} \mathrm{C}$ for $30 \mathrm{~s}$. Melting curves were generated to confirm the specificity of the amplification. Experiments were repeated in triplicate. Relative gene expression was calculated using the 2- $\triangle \triangle C$ t method. Primer sequences were as following. CXCL2: forward, 5'-CCA ACC ACC AGG CTA CAG G-3'; reverse, 5'-GCG TCA CAC TCA AGC TCT G-3'; GAPDH: forward 5'-ACC CAC TCC TCC ACC TTT GAC-3'; reverse, 5'-TGT TGC TGT AGC CAA ATT CGT T-3'.

\section{Oncomine database analysis}

The expression level of CXCL2 gene in various types of cancer was determined in Oncomine database (https://www.oncomine.org/). ${ }^{25}$ The threshold was determined according to the following values: $P$ value was 0.001 , fold change was 1.5 , and gene ranking of all.

\section{PrognoScan database analysis}

The correlation between CXCL2 expression and the survival rate of various types of cancer was analyzed by PrognoScan (http://dna00.bio.kyutech.ac.jp/PrognoScan/). ${ }^{26}$ Searching for the relationship between gene expression and patient prognosis in PrognoScan, such as overall survival (OS), relapse free survival (RFS) and disease free survival (DFS). Adjust the threshold value to Cox $P<0.05$.

\section{Kaplan-Meier plotter database analysis}

Kaplan-Meier plotter can assess the effect of $54 \mathrm{k}$ genes on survival in 21 cancer types. The datasets include 6,234 breast, 2,190 ovarian, 3,452 lung, and 1,440 gastric cancer. The system includes gene chip and RNA-seq data-sources for the databases include GEO, EGA, and TCGA. The correlation between CXCL2 expression and survival in breast, ovarian, lung and gastric cancers was analyzed by Kaplan-Meier plotter (http:// kmplot.com/analysis/). ${ }^{27}$ The hazard ratio (HR) with 95\% confidence intervals and log-rank $P$-value were also computed.

\section{TIMER database analysis}

TIMER is a comprehensive resource for systematical analysis of immune infiltrates across diverse cancer types (https://cistrome.shinyapps.io/timer/). ${ }^{28}$ The abundances of six immune infiltrates (B cells, CD4 + T cells, CD8 + T cells, Neutrophils, Macrophages, and Dendritic cells) are estimated by TIMER algorithm. We analyzed the expression of CXCL2 in different types of cancer and the correlation between CXCL2 expression and immune infiltration. In addition, the correlation between CXCL2 expression and gene markers of tumor infiltrating immune cells was explored. The correlation module generates a scatter plot of expression between a pair of user-defined genes in a given cancer type, together with the Spearman's correlation and the estimated statistical significance. CXCL2 is used for X-axis with gene symbol, and the related marker gene is expressed as gene symbol on Y-axis. The gene expression level was displayed by $\log 2$ RSEM.

\section{GEPIA analysis}

Gene Expression Profiling Interactive Analysis (GEPIA) (http://gepia.cancer-pku.cn/index.html) ${ }^{29}$ is used to further identify the significant related genes in TIMER GEPIA is a web-based tool for analyzing the RNA sequencing expression data of 9,736 tumors and 8,587 normal samples based on the TCGA and the GTEx data. GEPIA was used to generate survival curves including OS and DFS based on gene expression and log rank test and Mantel-Cox test for 33 different types of cancer. The correlation analysis of gene expression was carried out for a given TCGA expression data set. The correlation coefficient was determined by Spearman method. CXCL2 is used for the x-axis, and other genes of interest are expressed on the $y$-axis. Tumor and normal tissue data sets are used for analysis.

\section{Statistical analysis}

The survival curve was drawn by PrognoScan and Kaplan-Meier plotter. The results generated in Oncomine are shown as $P$ values, fold changes, and ranks. The results of Kaplan-Meier plotter, PrognoScan and GEPIA are displayed together with the HR and $P$ or Cox $P$-values of the log-rank test. The correlation of gene expression was evaluated by Spearman's correlation and statistical significance. $P<0.05$ was considered statistically significant.

\section{Results}

\section{Expression level of CXCL2 in different malignant tumors}

We analyzed CXCL2 expression level in cancer and normal tissues of a variety of malignant tumors through Oncomine database to clarify the expression difference. The results showed that the expression of CXCL2 in colorectal cancer, esophageal cancer, head and neck cancer, and lymphoma was higher than that in normal tissues. In other data sets, the expression of CXCL2 was lower in bladder cancer, BC, colorectal cancer, leukemia, lung cancer, liver cancer, lymphoma and sarcoma (Fig. 1A).

We further evaluated the expression of CXCL2 in cancer by TIMER database, and detected the expression of CXCL2 by RNA sequence data of various malignant tumors in TCGA. The differential expression of CXCL2 in all TCGA tumors is shown in Fig. 1B. Compared with adjacent normal tissues, CXCL2 expression was significantly lower in BLCA (bladder urothelial carcinoma), BRCA (breast invasive carcinoma), CHOL (cholangiocarcinoma), HNSC (head and 
neck cancer), $\mathrm{KICH}$ (kidney chromophobe), LIHC (liver hepatocellular carcinoma), LUAD (lung adenocarcinoma), LUSC (Lung squamous cell carcinoma), and PRAD (prostate adenocarcinoma), but it was significantly higher in COAD (colon adenocarcinoma), KIRC (kidney renal clear cell carcinoma), and READ (rectum adenocarcinoma).

\section{The role of CXCL2 in cancer prognosis evaluation}

We studied the correlation between the expression of CXCL2 and the prognosis of cancer patients. Using the PrognoScan, we evaluated the impact of CXCL2 expression to survival rates. It was remarkable that the expression of CXCL2 significantly impacts prognosis in colorectal, breast, lung, blood, eye and prostate cancers (Fig. 2A-Q). In colorectal cancer, three datasets (GSE17536 with 177 samples, GSE17537 with 55 samples and GSE14333 with 226 samples) ${ }^{30,31}$ showed that low CXCL2 expression was associated with poorer prognosis (OS HR=-3.26, $95 \% \mathrm{Cl}=0.01$ to $0.26, \mathrm{Cox} P=0.0008 ; 0 \mathrm{HR}=-4.26,95 \% \mathrm{Cl}=0.00$ to 0.20, Cox $P=0.0017$; DSS HR=-3.38, $95 \% \mathrm{Cl}=0.00$ to 0.32 , Cox $P=0.003$; DSS HR=-3.74, $95 \% \mathrm{Cl}=0.00$ to $0.57, \mathrm{Cox} P=0.02 ; \mathrm{DFS} \mathrm{HR}=-4.39,95 \% \mathrm{Cl}=0.00$ to 0.27 , $\operatorname{Cox} P=0.005$; DFS HR=-0.33, $95 \% \mathrm{Cl}=0.55$ to 0.94 , $\operatorname{Cox} P=0.017$; DFS HR=-2.79, $95 \% \mathrm{Cl}=0.00$ to $0.81, \mathrm{Cox} P=0.03 ; \mathrm{DFS} \mathrm{HR}=-0.39,95 \% \mathrm{Cl}=0.47$ to 0.98, Cox $P=0.037$ ) (Fig. 2A-H). In BC, two datasets (GSE3494-GPL96 with 236 samples and GSE1456-GPL96 with 159 samples) ${ }^{32,33}$ showed that low CXCL2

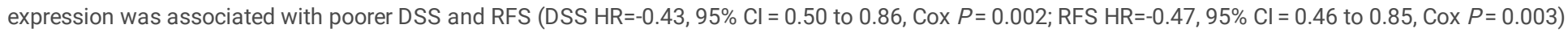
(Fig. 2l-J); dataset GSE3143 with 158 samples showed that high CXCL2 expression was associated with poorer OS (OS HR $=0.36,95 \% \mathrm{Cl}=1.07$ to $1.93, \mathrm{Cox} P$ $=0.017$ ) (Fig. 2K). In lung cancer, two datasets (GSE13213 with 117 samples and GSE31210 with 204 samples) ${ }^{34,35}$ showed that low CXCL2 expression was associated with poorer OS and RFS (OS HR=-0.29, 95\% Cl=0.61 to 0.92, Cox $P=0.005$; OS HR=-0.37, $95 \% \mathrm{Cl}=0.50$ to $0.95, \mathrm{Cox} P=0.024 ; \mathrm{RFS} \mathrm{HR}=-0.35,95 \%$ $\mathrm{Cl}=0.52$ to 0.95 , Cox $P=0.023$ ) (Fig. $2 \mathrm{~L}-\mathrm{N}$ ). Therefore, abnormal expression of CXCL2 is an independent risk factor for poor prognosis in colorectal, breast and lung cancer patients.

In order to further study the prognostic effect of CXCL2 on different cancers, Kaplan Meier plotter database was used to evaluate the prognostic value of CXCL2. The results showed that the poor prognosis of gastric cancer was related to the decreased expression of $\mathrm{CXCL2}(\mathrm{OS} \mathrm{HR}=0.62,95 \% \mathrm{Cl}=0.51$ to 0.75 , $P=1.1 \mathrm{e}-06$; PFS HR $=0.67,95 \% \mathrm{Cl}=0.53$ to $0.84, P=0.00047$ ) (Fig. $2 \mathrm{~T}, \mathrm{U}$ ), and the expression of CXCL2 had more significant effect on RFS of BC (PFS HR = $0.8,95 \% \mathrm{Cl}=0.72$ to $0.89, P=6 \mathrm{e}-05$ ) and $\mathrm{OS}$ of lung cancer (OS HR=0.87, 95\% $\mathrm{Cl}=0.77$ to $0.99, P=0.032)$ (Fig. $2 \mathrm{R}, \mathrm{S})$, but less effect on ovarian cancer. These results indicate that the expression of $\mathrm{CXCL} 2$ has an effect on the prognosis of gastric cancer, $\mathrm{BC}$ and lung cancer.

\section{Abnormal expression of CXCL2 affects the prognosis of gastric cancer and breast cancer patients with lymph node metastasis}

To better understand the potential mechanism of CXCL2 expression in cancer, we used the Kaplan-Meier plotter database to study the relationship between the expression of CXCL2 and the clinical characteristics of gastric and breast cancer patients.

In gastric cancer, the abnormal expression of CXCL2 was correlated with OS and PFS in male and female patients and moderate differentiation, and with OS in Lauren classification $(P<0.05)$. Especially, CXCL2 expression was correlated with OS and PFS in stage $3(0 S \mathrm{HR}=0.62, \mathrm{P}=0.0014 ; \mathrm{PFS} H \mathrm{HR}=0.58, P=$ 0.005 ), but not with OS and PFS of stage 1 to 2 and stage N1 patients (OS HR $=0.68, P=0.0792 ;$ PFS HR $=0.77, P=0.2025)($ Table 1 ). In addition, the abnormal expression of CXCL2 has the highest HR values of $\mathrm{N} 2$ in the $\mathrm{N}$ categories.

In $\mathrm{BC}$, we see more that the abnormal expression of CXCL2 has more effect on RFS, but in the lymph node status, we found that the expression of CXCL2 has significant effect on OS and RFS of lymph node positive patients (OS HR $=0.65, P=0.0335$; PFS HR $=0.76, P=0.0058)($ Table 2 ). These results suggest that CXCL2 expression level can impact the prognosis in gastric and breast cancer patient with lymph node metastasis.

\section{CXCL2 expression is correlated with immune infiltration in gastric and breast cancers}

Tumor-infiltrating lymphocytes are an independent predictor of sentinel lymph node status and survival in patients with cancers. ${ }^{36,37}$ Therefore, we further investigated whether the expression of CXCL2 was related to the levels of immune infiltration in different cancers. The purity of tumor is an important factor affecting the analysis of immune infiltration in clinical tumor samples, so we choose the cancer types whose expression level of CXCL2 is negatively correlated with the purity of tumor and is significantly correlated with the prognosis. We found that the expression of CXCL2 was correlated with better prognosis and high immune infiltration of BRCA. CXCL2 expression was positively correlated with the infiltration of CD8 $+\mathrm{T}$ cells $(r=0.098, P=2.10 \mathrm{e}-03)$, CD4 $+\mathrm{T}$ cells $(\mathrm{r}=0.173, P=6.30 \mathrm{e}-08)$, neutrophils $(\mathrm{r}=0.133, P=3.81 \mathrm{e}-05)$ and DCs $(r=0.115, P=3.73 \mathrm{e}-04)$ in BRCA (Fig. 3A). In STAD, there were negative correlations with infiltration of $B$ cells $(\mathrm{r}=-0.175, P=7.13 \mathrm{e}-04)$, CD $4+\mathrm{T}$ cells $(\mathrm{r}=-0.316, P=6.54 \mathrm{e}-10)$, macrophages $(\mathrm{r}=-0.238, P=3.54 \mathrm{e}-06)$, and $\mathrm{DCs}(\mathrm{r}=-0.185$, $P=3.37 \mathrm{e}-04$ ) (Fig. 3D). In COAD, CXCL2 expression has no significant correlations with tumor purity and infiltrating levels of B cells, CD8 $+\mathrm{T}$ cells, and dendritic cells (Fig. 3B). In addition, CXCL2 expression has no significant correlations with infiltrating levels of B cells, CD $8+T$ cells, CD4 + T cells, macrophages, and dendritic cells in LUAD (Fig. 3C).

Then, according to the different types of $\mathrm{BC}$, we further analyzed the correlation between the expression of CXCL2 and the immune infiltration of different types of BC. The results showed that in luminal BC, the expression of CXCL2 was positively correlated with the infiltration of CD8 $+\mathrm{T}$ cells $(\mathrm{r}=0.188, P=1.21 \mathrm{e}-$ 05), CD $4+\mathrm{T}$ cells $(\mathrm{r}=0.226, P=1.16 \mathrm{e}-07)$, macrophages $(\mathrm{r}=0.089, P=3.87 \mathrm{e}-02)$, neutrophils $(\mathrm{r}=0.146, P=7.04 \mathrm{e}-04)$ and DCS $(\mathrm{r}=0.136, P=1.61 \mathrm{e}-03)$ (Fig. 4D). In the other two types of BC, the expression of CXCL2 was not significantly correlated with immune infiltration. These findings suggest that CXCL2 plays a specific role in the immune infiltration of breast and gastric cancers, especially in BC.

\section{Relationship between CXCL2 expression and immune markers}

To further explore the relationship between CXCL2 and various immune infiltrating cells, we studied the relationship between CXCL2 and immune markers of different immune cells in BRCA, STAD and LUAD through TIMER and GEPIA databases (Table 3 and Table 4). We also analyzed T cells with different functions, such as Th1 cells, Th2 cells, Tfh cells, Th17 cells and Tregs, as well as exhausted T cells. After purity related adjustment, CXCL2 expression level 
was significantly correlated with most of the immune markers of different immune cells and different T cells in BRCA. However, CXCL2 expression level was significantly correlated with only 28 gene markers in STAD and 20 in LUAD (Table 3).

In addition, we found that the expression levels of most markers of monocytes, TAMs and M2 macrophage were strongly correlated with the expression of CXCL2 in BRCA (Table 3). We also found that CD86 of monocytes, CCL-2 and IL10 of TAMs, PTGS2 of M1 phenotype, CD163, VSIG4 and MS4A4A of M2 phenotype were significantly correlated with the expression of CXCL2 in BRCA $(P<0.05$; Fig. 5). We further analyzed the correlation between CXCL2 expression and the above markers in monocytes, TAMs and M2 macrophage by GEPIA database. The results between CXCL2 and markers of monocytes and TAMs in BRCA were similar to those in TIMER (Table 4). These suggested that CXCL2 may regulate the polarization of macrophages in BRCA.

The expression of CXCL2 was related to the infiltration level of Natural killer cell and DCS in BRCA, and the expression of Natural killer cell markers KIR2DL4, KIR3DL1 and KIR3DL2, as well as DC markers HLA-DPB1, HLA-DQB1, BDCA-1 and BDCA-4 were also significantly correlated with the expression of CXCL2 ( $P<$ 0.01 ) (Table 3). These results further reveal the close relationship between CXCL2 and the infiltration of Natural killer cell and DCS. At the same time, CXCL2 was significantly correlated with Treg and T cell exhaustion marker genes, such as CCR8, STAT5b, PD-1, CTLA4, LAG3 and GZMB. CCR8 plays an important role in Treg cells. PD-1, as one of the markers of T-cell exhaustion, is significantly related to the expression of CXCL2, suggesting that the expression of CXCL2 plays an important role in PD-1 mediated T-cell exhaustion. These results further confirm that CXCL2 is related to the immune infiltrating cells in BRCA,

suggesting that CXCL2 plays an important role in immune escape in BRCA microenvironment.

\section{CXCL2 expression is regulated by miR-532-5p}

The prediction results of StarBase database ${ }^{38}$ show that CXCL2 may be the target gene of miR-532-5p. Jiang and song et al respectively confirmed that CXCL2 is the target gene of miR-532-5p in HCC, and confirmed the combination of the two binding sites by double luciferase reporter gene system. ${ }^{39,40}$ Huang et al confirmed the abnormal expression of miR-532-5p in BC, and played a role as an oncogene. ${ }^{41}$ Our experimental results confirmed that after overexpression of miR-532-5p, the level of CXCL2 mRNA decreased, and after inhibition of miR-532-5p expression, the level of CXCL2 mRNA increased, with significant statistical significance (Fig. 6B,C). Then we analyzed the influence of miR-532-5p on the prognosis of patients with different clinicopathological characteristics by Kaplan-Meier plotter database (Fig. 6A and Table 5). The results showed that the expression of miR-532-5p was significantly correlated with HER2 negative $(P=0.0013)$, HER2 + ER- $(P=0.034)$ in molecular subtype and 2 and 3 Grades $(P=0.0396$ and $P=0.032)$. These results confirmed that CXCL2 was regulated by miR-532-5p in BC. MiR-532-5p has significant correlation with prognosis of patients in HER2 status, molecular subtype and grade.

\section{Discussion}

CXCL2 is a member of chemokine superfamily, which mainly comes from activated macrophages, ${ }^{42}$ and also known as growth-related gene product (GRO $\beta$ ). It has a strong chemotaxis effect on neutrophils ${ }^{43}$ and abnormal expression in a variety of tumor cells. Our analysis showed that CXCL2 expression level was related to the prognosis of different types of cancer. Low expression of CXCL2 is associated with poor prognosis in BC patients. CXCL2 expression level can affect the prognosis of patients with lymph node metastasis of gastric cancer and BC, indicating that CXCL2 expression can be used as a predictor of tumor metastasis. In addition, the analysis showed that the expression of CXCL2 was related to the level of immune infiltration and different immune marker sets in BC. Our research provides new insights into the potential role of CXCL2 in tumor immunity and its application as a biomarker of tumor.

In this study, we used different databases to detect the expression level and prognosis of CXCL2 in various types of cancer. The expression of CXCL2 was different in different cancer tissues and normal tissues. In the Oncomine database, the expression of CXCL2 in colorectal cancer, esophageal cancer, head and neck cancer, and lymphoma was higher than that in normal tissues. In other data sets, the expression of CXCL2 was lower in bladder cancer, BC, colorectal cancer, leukemia, lung cancer, liver cancer, lymphoma and sarcoma. But in TIMER database, CXCL2 expression was significantly lower in BLCA, BRCA, CHOL, HNSC, KICH, LIHC, LUAD, LUSC, and PRAD, but it was significantly higher in COAD, KIRC, and READ. The differences of CXCL2 expression levels in different types of cancer in different databases may reflect the data collection methods and potential mechanisms related to different biological characteristics. Analysis of data from the PrognoScan and Kaplan-Meier plotter databases showed that low expression of CXCL2 was associated with poor prognosis in colorectal, breast and lung cancer. In datasets of PrognoScan, Low expression of CXCL2 may be an independent risk factor for poor prognosis in colorectal cancer, BC and lung cancer. Kaplan-Meier plotter showed that the abnormal expression of CXCL2 was significantly related to the prognosis of gastric cancer, $\mathrm{BC}$ and lung cancer. These findings strongly suggest that CXCL2 is a prognostic biomarker for colorectal cancer, gastric cancer, $\mathrm{BC}$ and lung cancer.

Another important aspect of this study is that the expression of CXCL2 is associated with different levels of immune infiltration in BC. We found that the CXCL2 expression was positively correlated with the infiltration of CD8 + T cells, CD $4+T$ cells, neutrophils and DCs in BRCA. In STAD, there were negative correlations with infiltration of B cells, CD4 + T cells, macrophages, and DCs. Moreover,CXCL2 plays an important role in tumor immunomodulation of BRCA, we found that most markers of monocytes, TAMs and M2 macrophage were strongly correlated with the expression of CXCL2 in BRCA, such as the CD115 of monocytes, CCL-2 and IL10 of TAMs, CD163, VSIG4 and MS4A4A of M2 phenotype. These results reveal the potential regulatory role of CXCL2 in the polarization of tumor-associated macrophages. At the same time, CXCL2 was significantly correlated with Treg and T cell exhaustion marker genes, such as CCR8, STAT5b, PD-1, CTLA4, LAG3 and GZMB. In addition, there was a significant correlation between the expression of CXCL2 and the regulation of several markers of T helper cells in BRCA. These correlations may indicate the potential mechanism of CXCL2 regulating T cell function in BRCA. These results suggest that CXCL2 plays an important role in the recruitment and regulation of BRCA immune infiltrating cells.

We further confirmed that CXCL2 was regulated by miR-532-5p in BC. The expression of miR-532-5p was significantly correlated with HER2 negative, HER2 + ER - in molecular subtype and 2 and 3 Grades.

Anyway, the abnormal expression of CXCL2 is associated with poor prognosis and increased immune infiltration of CD8 + T cells, CD4 + T cells, macrophages, neutrophils and DCs in many cancers. In BC, the expression of CXCL2 may be involved in the regulation of tumor-associated macrophages (TAMs), $T$ cell 
exhaustion and Tregs, while CXCL2 as a target gene is regulated by miR-532-5p. Therefore, miR-532-5p may play an important role in immune cell infiltration and prognosis biomarkers of breast cancer patients by regulating CXCL2.

\section{Declarations}

\section{Ethics approval and consent to participate}

Not applicable

Consent for publication

Informed consent was obtained from all individual participants included in the study.

\section{Availability of data and materials}

Not applicable

\section{Competing interests}

The authors declare that they have no competing interests.

Funding

This work was supported by Taizhou Science and Technology Plan Project (No. 1901ky17)

\section{Authors' contributions}

JXA and FLC designed the experiments; YYC and ZSM performed the experiments; JXH and WJY collected the data; JXA analyzed the data; JXA wrote the manuscript. All authors read and approved the final manuscript.

Acknowledgements

Not applicable

\section{References}

1. Bray F, Ferlay J, Soerjomataram I, Siegel RL, Torre LA, Jemal A. Global cancer statistics 2018: GLOBOCAN estimates of incidence and mortality worldwide for 36 cancers in 185 countries. CA Cancer J Clin. 2018;68(6):394-424.

2. Gonzaga MA. How accurate is ultrasound in evaluating palpable breast masses? Pan Afr Med J. 2010;7:1.

3. Zhou S, He Y, Yang S, et al. The regulatory roles of IncRNAs in the process of breast cancer invasion and metastasis. Biosci Rep. 2018;38(5):BSR20180772.

4. Igney FH, Krammer PH. Immune escape of tumors: apoptosis resistance and tumor counterattack. J Leukoc Biol. 2002;71(6):907-20.

5. Borden ES, Kang P, Natri HM, et al. Neoantigen Fitness Model Predicts Lower Immune Recognition of Cutaneous Squamous Cell Carcinomas Than Actinic Keratoses. Front Immunol. 2019;10:2799.

6. Jiang X, Wang J, Deng X, et al. Role of the tumor microenvironment in PD-L1/PD-1-mediated tumor immune escape. Mol Cancer. 2019;18(1):10.

7. Rieth J, Subramanian S. Mechanisms of Intrinsic Tumor Resistance to Immunotherapy. Int J Mol Sci. 2018;19(5):1340.

8. Emens LA. Breast Cancer Immunotherapy: Facts and Hopes. Clin Cancer Res. 2018;24(3):511-20.

9. Salgado R, Denkert C, Demaria S, et al. The evaluation of tumor-infiltrating lymphocytes (TILs) in breast cancer: recommendations by an International TILs Working Group 2014. Ann Oncol. 2015;26(2):259-71.

10. Baggiolini M, Loetscher P, Moser B. Interleukin-8 and the chemokine family. Int J Immunopharmacol. 1995;17:103-8.

11. Matzer SP, Rodel F, Strieter RM, Rollinghoff M, Beuscher HU. Constitutive expression of CXCL2/MIP-2 is restricted to a Gr-1high, CD11b+, CD62Lhigh subset of bone marrow derived granulocytes. Int Immunol. 2004;16:1675-83.

12. Mantovani A, Sozzani S, Locati M, Allavena P, Sica A. Macrophage polarization: tumorassociated macrophages as a paradigm for polarized M2 mononuclear phagocytes. Trends Immunol. 2002;23:549-55.

13. Reutershan J, Ley K. Bench-to-bedside review: acute respiratory distress syndrome - how neutrophils migrate into the lung. Crit Care. 2004;8(6):453-61.

14. Hosoki K, Itazawa T, Boldogh I, Sur S. Neutrophil recruitment by allergens contribute to allergic sensitization and allergic inflammation. Curr Opin Allergy Clin Immunol. 2016;16(1):45-50.

15. Song XF, Wang Z, Jin Y, Wang Y, Duan WB. Loss of miR-532-5p in vitro promotes cell proliferation and metastasis by influencing CXCL2 expression in HCC. Am J Transl Res. 2015;7(11):2254-61.

16. Gao Y, Guan ZF, Chen JQ, et al. CXCL5/CXCR2 axis promotes bladder cancer cell migration and invasion by activating PI3K/AKT-induced upregulation of MMP2/MMP9. Int J Oncol. 2015;47(2):690-700.

17. Al-Alwan LA, Chang Y, Mogas A, et al. Differential roles of CXCL2 and CXCL3 and their receptors in regulating normal and asthmatic airway smooth muscle cell migration. J Immunol. 2013;191(5):2731-41.

18. Natsume M, Shimura T, Iwasaki $\mathrm{H}$, et al. Omental adipocytes promote peritoneal metastasis of gastric cancer through the CXCL2-VEGFA axis. Br $\mathrm{J}$ Cancer. 2020. 10.1038/s41416-020-0898-3. 
19. Song X, Wang Z, Jin Y, Wang Y, Duan W. Loss of miR-532-5p in vitro promotes cell proliferation and metastasis by influencing CXCL2 expression in HCC. Am J Transl Res. 2015;7(11):2254-61.

20. Kavandi L, Collier MA, Nguyen H, et al. Progesterone and calcitriol attenuate inflammatory cytokines CXCL1 and CXCL2 in ovarian and endometrial cancer cells. Journal of cellular biochemistry. 2012;113(10):3143-52.

21. Taki M, Abiko K, Baba T, et al. Snail promotes ovarian cancer progression by recruiting myeloid-derived suppressor cells via CXCR2 ligand upregulation. Nat Commun. 2018;9(1):1685.

22. He C, Carter AB. The Metabolic Prospective and Redox Regulation of Macrophage Polarization. J Clin Cell Immunol. 2015;6(6):371.

23. Yahata T, Mizoguchi M, Kimura A, et al. Programmed cell death ligand 1 disruption by clustered regularly interspaced short palindromic repeats/Cas9genome editing promotes antitumor immunity and suppresses ovarian cancer progression. Cancer Sci. 2019;110(4):1279-92.

24. Owen JL, Criscitiello MF, Libreros S, et al. Expression of the inflammatory chemokines CCL2, CCL5 and CXCL2 and the receptors CCR1-3 and CXCR2 in T lymphocytes from mammary tumor-bearing mice. Cell Immunol. 2011;270(2):172-82.

25. Rhodes DR, Kalyana-Sundaram S, Mahavisno V, et al. Oncomine 3.0: genes, pathways, and networks in a collection of 18,000 cancer gene expression profiles. Neoplasia. 2007;9(2):166-80.

26. Mizuno H, Kitada K, Nakai K, Sarai A. PrognoScan: a new database for meta-analysis of the prognostic value of genes. BMC Med Genomics. 2009;2:18.

27. Nagy Á, Lánczky A, Menyhárt O, Győrffy B. Validation of miRNA prognostic power in hepatocellular carcinoma using expression data of independent datasets [published correction appears in Sci Rep. 2018 Jul 26;8(1):11515]. Sci Rep. 2018; 8(1):9227.

28. Li T, Fan J, Wang B, et al. TIMER: A Web Server for Comprehensive Analysis of Tumor-Infiltrating Immune Cells. Cancer Res. 2017;77(21):e108-10.

29. Tang Z, Li C, Kang B, Gao G, Li C, Zhang Z. GEPIA: a web server for cancer and normal gene expression profiling and interactive analyses. Nucleic Acids Res. 2017;45(W1):W98-102.

30. Smith JJ, Deane NG, Wu F, et al. Experimentally derived metastasis gene expression profile predicts recurrence and death in patients with colon cancer. Gastroenterology. 2010;138(3):958-68.

31. Jorissen RN, Gibbs P, Christie M, et al. Metastasis-Associated Gene Expression Changes Predict Poor Outcomes in Patients with Dukes Stage B and C Colorectal Cancer. Clin Cancer Res. 2009;15(24):7642-51.

32. Miller LD, Smeds J, George J, et al. An expression signature for p53 status in human breast cancer predicts mutation status, transcriptional effects, and patient survival [published correction appears in Proc Natl Acad Sci U S A. 2005 Dec 6; 102(49):17882]. Proc Natl Acad Sci U S A. 2005; 102(38):1355013555.

33. Pawitan Y, Bjöhle J, Amler L, et al. Gene expression profiling spares early breast cancer patients from adjuvant therapy: derived and validated in two population-based cohorts. Breast Cancer Res. 2005;7(6):R953-64.

34. Tomida S, Takeuchi T, Shimada Y, et al. Relapse-related molecular signature in lung adenocarcinomas identifies patients with dismal prognosis. J Clin Oncol. 2009;27(17):2793-9.

35. Okayama H, Kohno T, Ishii Y, et al. Identification of genes upregulated in ALK-positive and EGFR/KRAS/ALK-negative lung adenocarcinomas. Cancer Res. 2012;72(1):100-11.

36. Ohtani H. Focus on TILs: prognostic significance of tumor infiltrating lymphocytes in human colorectal cancer. Cancer Immun. 2007;7:4.

37. Azimi F, Scolyer RA, Rumcheva P, et al. Tumor-infiltrating lymphocyte grade is an independent predictor of sentinel lymph node status and survival in patients with cutaneous melanoma. J Clin Oncol. 2012;30(21):2678-83.

38. Li JH, Liu S, Zhou H, Qu LH, Yang JH. starBase v2.0: decoding miRNA-ceRNA, miRNA-ncRNA and protein-RNA interaction networks from large-scale CLIPSeq data. Nucleic Acids Res. 2014;42(Database issue):D92-7.

39. Jiang ZT, Han Y, Liu XY, Lv LY, Pan JH, Liu CD. Tripterine Restrains the Aggressiveness of Hepatocellular Carcinoma Cell via Regulating miRNA-5325p/CXCL2 Axis. Onco Targets Ther. 2020;13:2973-85.

40. Song X, Wang Z, Jin Y, Wang Y, Duan W. Loss of miR-532-5p in vitro promotes cell proliferation and metastasis by influencing CXCL2 expression in HCC. Am J Transl Res. 2015;7(11):2254-61.

41. Huang L, Tang X, Shi X, Su L. miR-532-5p promotes breast cancer proliferation and migration by targeting RERG. Exp Ther Med. 2020;19(1):400-8.

42. Widmer U, Manogue KR, Cerami A, Sherry B. Genomic cloning and promoter analysis of macrophage inflammatory protein (MIP)-2, MIP-1 alpha, and MIP1 beta, members of the chemokine superfamily of proinflammatory cytokines. J Immunol. 1993;150(11):4996-5012.

43. Inoue A, Matsumoto I, Tanaka Y, et al. TIARP attenuates autoantibody-mediated arthritis via the suppression of neutrophil migration by reducing CXCL2/CXCR2 and IL-6 expression. Sci Rep. 2016;6:38684.

\section{Tables}

Table 1. The relationship between CXCL2 expression and clinical prognosis in gastric cancer of different clinicopathological factors was detected by KaplanMeier plotter. 


\begin{tabular}{|c|c|c|c|c|c|c|}
\hline & \multicolumn{3}{|c|}{ Overall survival $(n=882)$} & \multicolumn{3}{|c|}{ Progression-free survival $(n=646)$} \\
\hline \multicolumn{7}{|l|}{ Parameter } \\
\hline & $\mathrm{N}$ & Hazard ratio & $P$-value & $\mathrm{N}$ & Hazard ratio & $P$-value \\
\hline \multicolumn{7}{|l|}{ Gender } \\
\hline Female & 236 & $0.44(0.29-0.67)$ & $6.6 \mathrm{e}-5$ & 201 & $0.52(0.34-0.8)$ & 0.0027 \\
\hline Male & 545 & $0.63(0.5-0.78)$ & $1.8 \mathrm{e}-5$ & 438 & $0.69(0.53-0.9)$ & 0.0061 \\
\hline \multicolumn{7}{|l|}{ Stage } \\
\hline 1 & 67 & $0.37(0.13-1.09)$ & 0.0605 & 60 & $0.56(0.18-1.78)$ & 0.3204 \\
\hline 2 & 140 & $0.56(0.28-1.11)$ & 0.0906 & 131 & $0.62(0.34-1.14)$ & 0.1207 \\
\hline 3 & 305 & $0.62(0.46-0.83)$ & 0.0014 & 186 & $0.58(0.4-0.85)$ & 0.005 \\
\hline 4 & 148 & $0.55(0.37-0.82)$ & 0.0026 & 141 & $1.23(0.83-1.82)$ & 0.2919 \\
\hline \multicolumn{7}{|l|}{ Stage T } \\
\hline 2 & 241 & $0.81(0.49-1.35)$ & 0.4225 & 239 & $1.35(0.89-2.04)$ & 0.1554 \\
\hline 3 & 204 & $0.66(0.47-0.94)$ & 0.0194 & 204 & $0.79(0.56-1.1)$ & 0.165 \\
\hline 4 & 38 & $0.34(0.13-0.88)$ & 0.0207 & 39 & $0.49(0.2-1.19)$ & 0.1081 \\
\hline \multicolumn{7}{|l|}{ Stage N } \\
\hline 0 & 74 & $0.34(0.13-0.89)$ & 0.0218 & 72 & $0.36(0.14-0.93)$ & 0.0274 \\
\hline 1 & 225 & $0.68(0.44-1.05)$ & 0.0792 & 222 & $0.77(0.51-1.15)$ & 0.2025 \\
\hline 2 & 121 & $0.45(0.28-0.71)$ & 0.0005 & 125 & $0.56(0.36-0.86)$ & 0.0077 \\
\hline 3 & 76 & $0.47(0.27-0.81)$ & 0.0058 & 76 & $0.6(0.34-1.06)$ & 0.0771 \\
\hline $1+2+3$ & 422 & $0.61(0.46-0.82)$ & 0.0007 & 423 & $0.69(0.53-0.91)$ & 0.0085 \\
\hline \multicolumn{7}{|l|}{ Stage M } \\
\hline 0 & 444 & $0.61(0.46-0.82)$ & 0.0008 & 443 & $0.7(0.53-0.92)$ & 0.0102 \\
\hline 1 & 56 & $0.5(0.26-0.95)$ & 0.0319 & 56 & $0.76(0.4-1.43)$ & 0.3905 \\
\hline \multicolumn{7}{|c|}{ Lauren classification } \\
\hline Intestinal & 320 & $0.56(0.4-0.79)$ & 0.0007 & 263 & $0.7(0.49-1.01)$ & 0.0553 \\
\hline Diffuse & 241 & $0.69(0.48-0.97)$ & 0.033 & 231 & $0.72(0.51-1.02)$ & 0.064 \\
\hline Mixed & 32 & $0.28(0.08-0.99)$ & 0.0352 & 28 & $2.5(0.83-7.53)$ & 0.094 \\
\hline \multicolumn{7}{|c|}{ Differentiation } \\
\hline Poor & 165 & $1.26(0.81-1.96)$ & 0.3065 & 121 & $1.4(0.83-2.36)$ & 0.2012 \\
\hline Moderate & 67 & $0.37(0.19-0.72)$ & 0.0027 & 67 & $0.45(0.23-0.85)$ & 0.0124 \\
\hline
\end{tabular}

The $P$-values shown in bold indicate $P<0.05$.

Table 2. The relationship between CXCL2 expression and clinical prognosis in breast cancer of different clinicopathological factors was detected by KaplanMeier plotter. 


\begin{tabular}{|c|c|c|c|c|c|c|}
\hline & \multicolumn{3}{|c|}{ Overall survival $(n=1402)$} & \multicolumn{3}{|c|}{ Relapse -free survival $(n=3955)$} \\
\hline \multicolumn{7}{|l|}{ Parameter } \\
\hline & $\mathrm{N}$ & Hazard ratio & $P$-value & $\mathrm{N}$ & Hazard ratio & $P$-value \\
\hline \multicolumn{7}{|l|}{ ER status } \\
\hline Positive & 548 & $0.7(0.49-1)$ & 0.0512 & 2061 & $0.71(0.6-0.83)$ & $3.4 \mathrm{e}-5$ \\
\hline Negative & 251 & $0.93(0.59-1.46)$ & 0.7455 & 801 & $1.11(0.89-1.39)$ & 0.36 \\
\hline \multicolumn{7}{|l|}{ PR status } \\
\hline Positive & 83 & $0.72(0.19-2.69)$ & 0.6268 & 589 & $0.62(0.43-0.89)$ & 0.0097 \\
\hline Negative & 89 & $1.1(0.44-2.76)$ & 0.8452 & 549 & $0.88(0.66-1.18)$ & 0.3914 \\
\hline \multicolumn{7}{|l|}{ HER2 status } \\
\hline Positive & 129 & $1.17(0.58-2.34)$ & 0.6594 & 252 & $1.06(0.69-1.64)$ & 0.7879 \\
\hline Negative & 130 & $0.72(0.3-1.72)$ & 0.4603 & 800 & $0.67(0.51-0.87)$ & 0.0029 \\
\hline \multicolumn{7}{|l|}{ Intrinsic subtype } \\
\hline Basal & 241 & $0.92(0.57-1.51)$ & 0.7504 & 618 & $0.96(0.74-1.23)$ & 0.7299 \\
\hline Luminal A & 611 & $0.82(0.57-1.17)$ & 0.2631 & 1933 & $0.67(0.56-0.79)$ & $4.4 \mathrm{e}-6$ \\
\hline Luminal B & 433 & $0.72(0.5-1.06)$ & 0.0917 & 1149 & $0.72(0.59-0.87)$ & 0.0009 \\
\hline HER2+ & 117 & $1.36(0.71-2.61)$ & 0.3491 & 251 & $1(0.68-1.47)$ & 0.9983 \\
\hline \multicolumn{7}{|l|}{ Grade } \\
\hline 1 & 161 & $1.13(0.44-2.93)$ & 0.8005 & 345 & $0.98(0.58-1.65)$ & 0.9447 \\
\hline 2 & 387 & $0.73(0.47-1.13)$ & 0.1622 & 901 & $0.89(0.7-1.13)$ & 0.346 \\
\hline 3 & 503 & $0.9(0.65-1.25)$ & 0.5365 & 903 & $0.9(0.72-1.12)$ & 0.3413 \\
\hline \multicolumn{7}{|l|}{ Lymph node status } \\
\hline Positive & 313 & $0.65(0.44-0.97)$ & 0.0335 & 1133 & $0.76(0.62-0.92)$ & 0.0058 \\
\hline Negative & 594 & $1.06(0.73-1.54)$ & 0.7437 & 2020 & $0.92(0.77-1.09)$ & 0.3131 \\
\hline \multicolumn{7}{|l|}{ TP53 status } \\
\hline Mutated & 111 & $0.46(0.21-1.03)$ & 0.0536 & 188 & $0.58(0.36-0.94)$ & 0.0254 \\
\hline Wild type & 187 & $0.65(0.34-1.25)$ & 0.1915 & 273 & $0.8(0.53-1.22)$ & 0.3036 \\
\hline \multicolumn{7}{|l|}{ Pietenpol subtype } \\
\hline Basal-like 1 & 58 & $0.87(0.29-2.59)$ & 0.8025 & 171 & $0.69(0.42-1.11)$ & 0.1257 \\
\hline Basal-like 2 & 38 & $3.02(0.78-11.7)$ & 0.0937 & 76 & $1.37(0.67-2.79)$ & 0.3901 \\
\hline Immunomodulatory & 100 & $1.89(0.71-5.04)$ & 0.1966 & 203 & $1.35(0.75-2.44)$ & 0.3136 \\
\hline Mesenchymal & 73 & $0.86(0.69-1.07)$ & 0.1692 & 177 & $1.12(0.73-1.72)$ & 0.5894 \\
\hline Mesenchymal stem-like & 19 & / & l & 63 & $1.21(0.55-2.66)$ & 0.6345 \\
\hline Luminal androgen receptor & 83 & $1.07(0.55-2.11)$ & 0.8373 & 203 & $0.97(0.65-1.46)$ & 0.9002 \\
\hline
\end{tabular}

The $P$-values shown in bold indicate $P<0.05$.

Table 3. Correlation analysis between CXCL2 and relate genes and markers of immune cells in TIMER. 


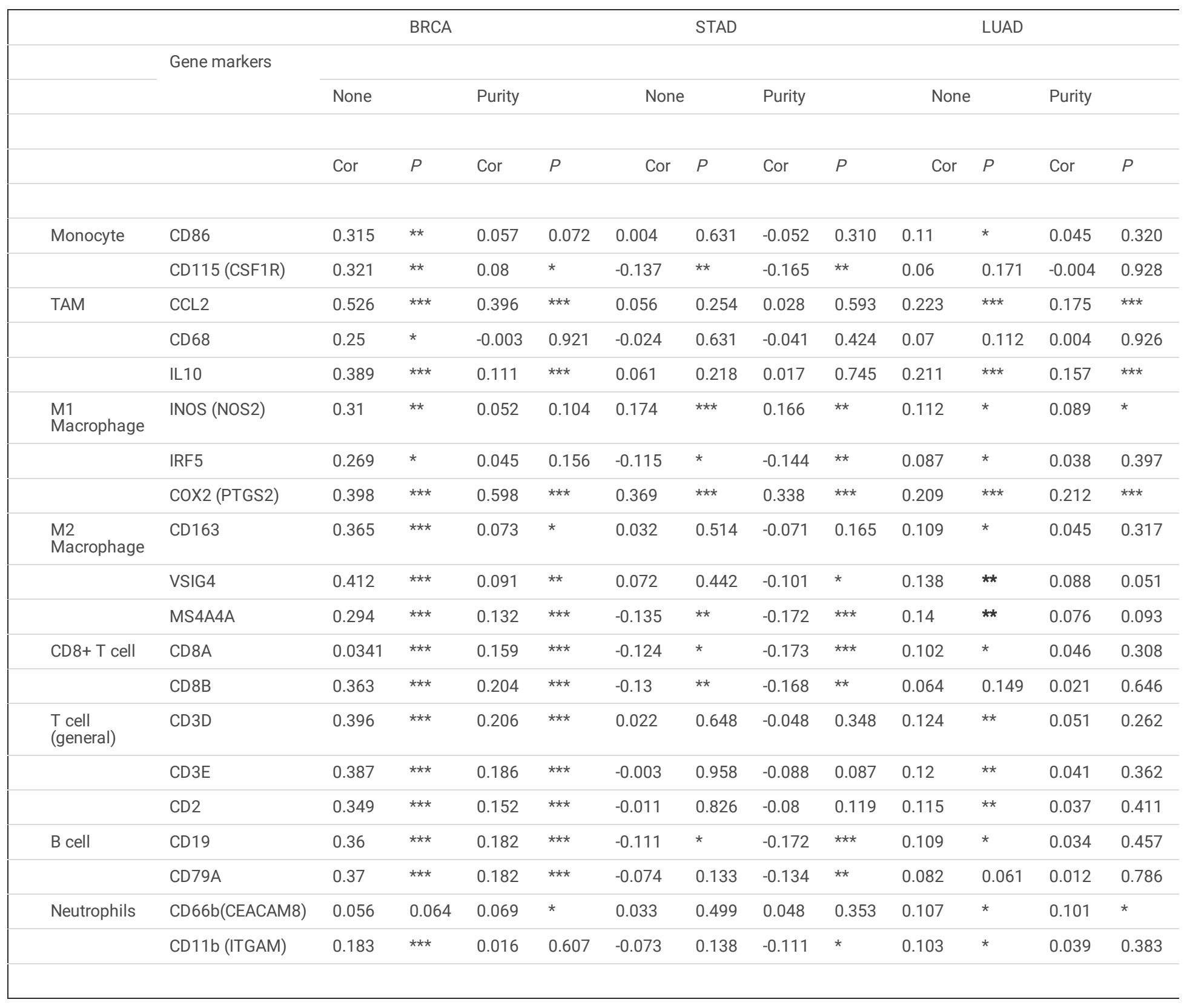




\begin{tabular}{|c|c|c|c|c|c|c|c|c|c|c|c|c|c|}
\hline & CCR7 & 0.408 & $\star \star \star$ & 0.225 & $\star \star \star ~$ & -0.101 & * & -0.177 & $\star * *$ & 0.168 & $\star \star \star *$ & 0.091 & * \\
\hline \multirow[t]{7}{*}{ Natural killer cell } & KIR2DL1 & 0.167 & $\star \star \star$ & 0.068 & * & 0.042 & 0.398 & 0.041 & 0.431 & 0.126 & ** & 0.105 & * \\
\hline & KIR2DL3 & 0.15 & $\star \star \star$ & 0.038 & 0.234 & 0.064 & 0.194 & 0.092 & 0.074 & 0.094 & * & 0.061 & 0.173 \\
\hline & KIR2DL4 & 0.221 & $\star \star \star$ & 0.11 & $\star * *$ & 0.164 & $\star \star \star$ & 0.116 & * & 0.06 & 0.171 & 0.039 & 0.393 \\
\hline & KIR3DL1 & 0.226 & $\star \star \star$ & 0.103 & $\star \star$ & 0.036 & 0.471 & 0.009 & 0.867 & 0.181 & $\star \star \star \star$ & 0.152 & $\star \star \star \star$ \\
\hline & KIR3DL2 & 0.224 & $\star * \star$ & 0.09 & ** & 0.072 & 0.141 & 0.062 & 0.231 & -0.001 & 0.977 & -0.037 & 0.410 \\
\hline & KIR3DL3 & 0.101 & $\star \star * *$ & 0.051 & 0.109 & 0.067 & 0.175 & 0.114 & * & 0.067 & 0.130 & 0.059 & 0.187 \\
\hline & KIR2DS4 & 0.178 & $\star \star \star *$ & 0.065 & * & 0.082 & 0.097 & 0.071 & 0.166 & 0.05 & 0.260 & 0.025 & 0.581 \\
\hline \multirow[t]{7}{*}{ Dendritic cell } & HLA-DPB1 & 0.34 & $\star \star \star *$ & 0.131 & 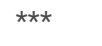 & -0.01 & 0.844 & -0.076 & 0.142 & 0.117 & ** & 0.059 & 0.190 \\
\hline & HLA-DQB1 & 0.269 & $\star \star \star$ & 0.122 & $\star \star *$ & 0.057 & 0.246 & 0 & 0.998 & 0.083 & 0.061 & 0.024 & 0.593 \\
\hline & HLA-DRA & 0.288 & $\star \star \star$ & 0.086 & $\star \star$ & 0.065 & 0.187 & 0.016 & 0.749 & 0.12 & ** & 0.061 & 0.174 \\
\hline & HLA-DPA1 & 0.261 & $\star * \star$ & 0.05 & 0.114 & 0.02 & 0.686 & 0.032 & 0.535 & 0.088 & * & 0.03 & 0.506 \\
\hline & BDCA-1(CD1C) & 0.467 & $\star \star \star *$ & 0.292 & $\star \star \star$ & -0.182 & $\star \star * *$ & -0.26 & $\star \star \star *$ & 0.235 & $\star \star \star *$ & 0.195 & $\star \star \star *$ \\
\hline & BDCA-4(NRP1) & 0.26 & $\star \star \star *$ & 0.127 & $\star \star \star$ & -0.071 & 0.149 & -0.116 & * & 0.093 & * & 0.081 & 0.074 \\
\hline & CD11c (ITGAX) & 0.271 & $\star \star *$ & 0.083 & ** & 0.028 & 0.564 & -0.001 & 0.991 & 0.216 & $\star \star \star$ & 0.163 & $\star * *$ \\
\hline \multirow[t]{5}{*}{ Th1 } & T-bet (TBX21) & 0.39 & $\star * *$ & 0.211 & $\star \star \star$ & -0.021 & 0.672 & -0.082 & 0.113 & 0.132 & $\star \star$ & 0.061 & 0.177 \\
\hline & STAT4 & 0.399 & $\star \star \star *$ & 0.213 & $\star \star \star *$ & -0.045 & 0.358 & -0.103 & * & 0.179 & $\star \star * *$ & 0.123 & ** \\
\hline & STAT1 & -0.001 & 0.976 & -0.098 & ** & 0.018 & 0.715 & 0.008 & 0.871 & -0.052 & 0.24 & -0.105 & * \\
\hline & IFN-y(IFNG) & 0.26 & $\star \star \star$ & 0.122 & $\star \star \star ~$ & 0.167 & $\star \star \star *$ & 0.122 & * & 0.043 & 0.328 & -0.005 & 0.907 \\
\hline & TNF-a (TNF) & 0.266 & $* \star \star$ & 0.2 & $\star \star \star *$ & 0.423 & $\star \star \star *$ & 0.382 & $\star \star \star$ & 0.199 & $\star \star \star *$ & 0.139 & ** \\
\hline \multirow[t]{4}{*}{ Th2 } & GATA3 & 0.338 & $\star \star \star *$ & -0.252 & 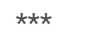 & -0.236 & $\star \star \star *$ & -0.295 & $\star \star \star \star ~$ & -0.046 & 0.294 & -0.134 & ** \\
\hline & STAT6 & 0.136 & $* \star \star$ & 0.081 & * & -0.122 & * & -0.121 & * & 0.138 & ** & 0.133 & ** \\
\hline & STAT5A & 0.406 & $\star * \star$ & 0.307 & $\star \star \star *$ & -0.102 & * & -0.136 & ** & 0.044 & 0.314 & -0.035 & 0.439 \\
\hline & IL13 & 0.256 & $\star * *$ & 0.212 & $\star \star \star$ & 0.058 & 0.238 & 0.067 & 0.194 & 0.181 & $\star \star \star *$ & 0.142 & ** \\
\hline \multirow[t]{2}{*}{ Tfh } & BCL6 & 0.17 & $* * *$ & 0.111 & $\star \star \star *$ & -0.057 & 0.246 & -0.112 & * & 0.174 & $\star \star *$ & 0.19 & $\star \star \star *$ \\
\hline & IL21 & 0.123 & $\star \star \star$ & 0.008 & 0.792 & 0.045 & 0.360 & 0.009 & 0.858 & -0.026 & 0.562 & -0.053 & 0.238 \\
\hline
\end{tabular}

\begin{tabular}{|c|c|c|c|c|c|c|c|c|c|c|c|c|c|}
\hline \multirow[t]{2}{*}{ Th17 } & STAT3 & 0.079 & ** & 0.012 & 0.712 & 0.013 & 0.796 & -0.014 & 0.788 & -0.009 & 0.834 & 0.003 & 0.938 \\
\hline & IL17A & 0.203 & $\star \star *$ & 0.155 & $\star \star \star *$ & 0.394 & $\star * *$ & 0.359 & $\star \star \star$ & 0.116 & ** & 0.1 & * \\
\hline \multirow[t]{4}{*}{ Treg } & FOXP3 & 0.181 & $\star \star \star \star$ & 0.003 & 0.931 & 0.01 & 0.839 & -0.032 & 0.528 & 0.021 & 0.634 & -0.07 & 0.121 \\
\hline & CCR8 & 0.057 & 0.058 & -0.082 & ** & -0.021 & 0.667 & -0.051 & 0.325 & 0.07 & 0.115 & 0.007 & 0.878 \\
\hline & STAT5B & 0.172 & $\star \star \star *$ & 0.116 & ** & -0.27 & $\star \star \star *$ & -0.294 & $\star \star \star$ & 0.021 & 0.638 & 0.012 & 0.787 \\
\hline & TGF $\beta$ (TGFB1) & 0.199 & $\star \star *$ & 0.03 & 0.352 & -0.178 & $* \star *$ & -0.222 & $* * *$ & 0.03 & 0.493 & -0.019 & 0.682 \\
\hline \multirow[t]{5}{*}{ T cell exhaustion } & PD-1 (PDCD1) & 0.38 & $\star \star \star$ & 0.211 & $\star \star \star$ & -0.075 & 0.127 & -0.116 & * & 0.068 & 0.122 & 0.002 & 0.958 \\
\hline & CTLA4 & 0.328 & 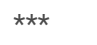 & 0.168 & $\star \star \star *$ & 0.092 & 0.061 & 0.047 & 0.361 & 0.232 & $* * *$ & 0.18 & $\star * *$ \\
\hline & LAG3 & 0.229 & $\star \star \star *$ & 0.13 & $\star \star \star *$ & 0.038 & 0.409 & -0.018 & 0.726 & 0.012 & 0.780 & -0.037 & 0.414 \\
\hline & TIM-3 (HAVCR2) & 0.168 & $\star \star \star$ & 0.003 & 0.935 & -0.014 & 0.777 & -0.057 & 0.267 & 0.126 & $\star \star$ & 0.056 & 0.218 \\
\hline & GZMB & 0.399 & $* \star *$ & 0.263 & $\star \star \star *$ & 0.201 & $\star \star \star *$ & 0.162 & ** & 0.136 & ** & 0.096 & * \\
\hline
\end{tabular}

BRCA, breast invasive carcinoma; STAD, stomach adenocarcinoma; LUAD, lung adenocarcinoma. TAM, Tumor-associated macrophages. Tumor, correlation analysis in tumor tissue of TCGA. Normal, correlation analysis in normal tissue of TCGA. ${ }^{\star} P<0.05 ; * * P<0.01 ; * * * P<0.001$. 
Table 4. Correlation analysis between CXCL2 and relate genes and markers of monocyte and macrophages in GEPIA.

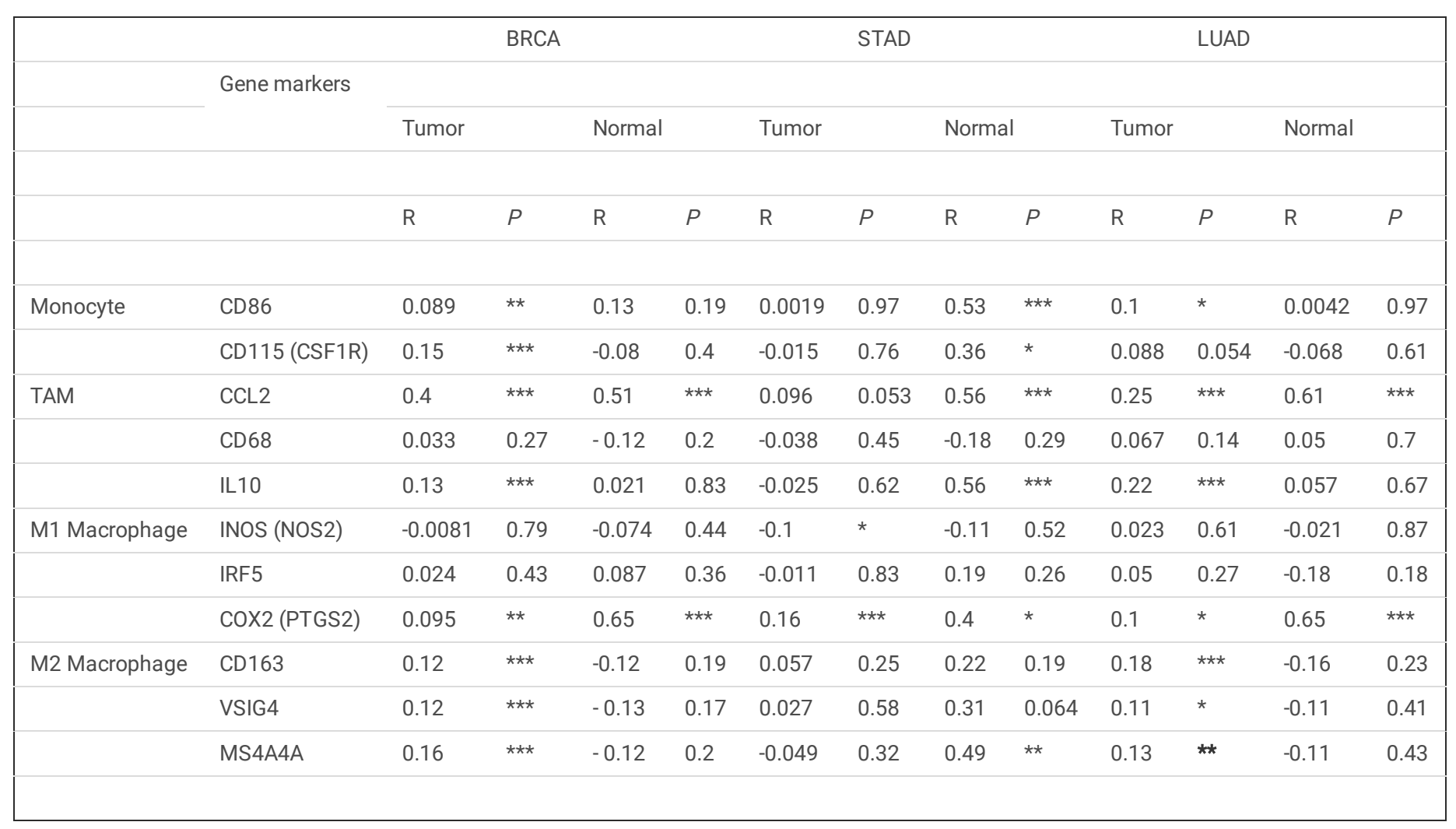

BRCA, breast invasive carcinoma; STAD, stomach adenocarcinoma; LUAD, lung adenocarcinoma. TAM, Tumor-associated macrophages. Tumor, correlation analysis in tumor tissue of TCGA. Normal, correlation analysis in normal tissue of TCGA. ${ }^{*} P<0.05 ; * * P<0.01 ; * * * P<0.001$

Table 5. The relationship between miR-532-5p expression and clinical prognosis in breast cancer of different clinicopathological factors was detected by Kaplan-Meier plotter. 


\begin{tabular}{|c|c|c|c|}
\hline & \multicolumn{3}{|c|}{ METABRIC overall survival $(n=1262)$} \\
\hline \multicolumn{4}{|l|}{ Parameter } \\
\hline & $\mathrm{N}$ & Hazard ratio & $P$-value \\
\hline \multicolumn{4}{|l|}{ ER status } \\
\hline \multicolumn{4}{|l|}{ IHC: } \\
\hline Positive & 996 & $1.35(1.05-1.73)$ & 0.018 \\
\hline Negative & 266 & $0.66(0.44-0.99)$ & 0.041 \\
\hline \multicolumn{4}{|l|}{ Gene chip: } \\
\hline Positive & 979 & $1.27(0.99-1.63)$ & 0.063 \\
\hline Negative & 283 & $0.74(0.5-1.09)$ & 0.13 \\
\hline \multicolumn{4}{|c|}{ HER2 status } \\
\hline \multicolumn{4}{|l|}{ Gene chip: } \\
\hline Positive & 157 & $0.64(0.39-1.04)$ & 0.068 \\
\hline Negative & 1105 & $1.45(1.16-1.83)$ & 0.0013 \\
\hline \multicolumn{4}{|c|}{ Molecular subtype } \\
\hline TNBC & 203 & $0.78(0.49-1.26)$ & 0.32 \\
\hline Luminal A & 546 & $1.37(0.95-1.99)$ & 0.091 \\
\hline Luminal B & 433 & $1.33(0.97-1.84)$ & 0.08 \\
\hline HER2+ER- & 80 & $0.49(0.25-0.96)$ & 0.034 \\
\hline \multicolumn{4}{|l|}{ Grade } \\
\hline 1 & 105 & $0.55(0.22-1.35)$ & 0.18 \\
\hline 2 & 490 & $1.44(1.02-2.04)$ & 0.0396 \\
\hline 3 & 620 & $0.74(0.57-0.98)$ & 0.032 \\
\hline \multicolumn{4}{|c|}{ Lymph node status } \\
\hline Positive & 585 & $0.75(0.57-0.99)$ & 0.0397 \\
\hline Negative & 672 & $1.47(1.05-2.06)$ & 0.025 \\
\hline
\end{tabular}

The $P$-values shown in bold indicate $P<0.05$.

\section{Figures}


A

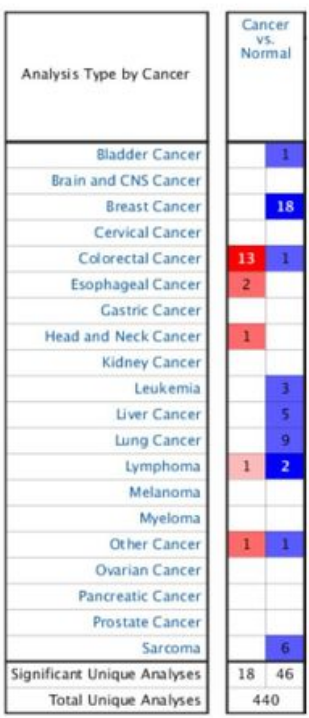

B

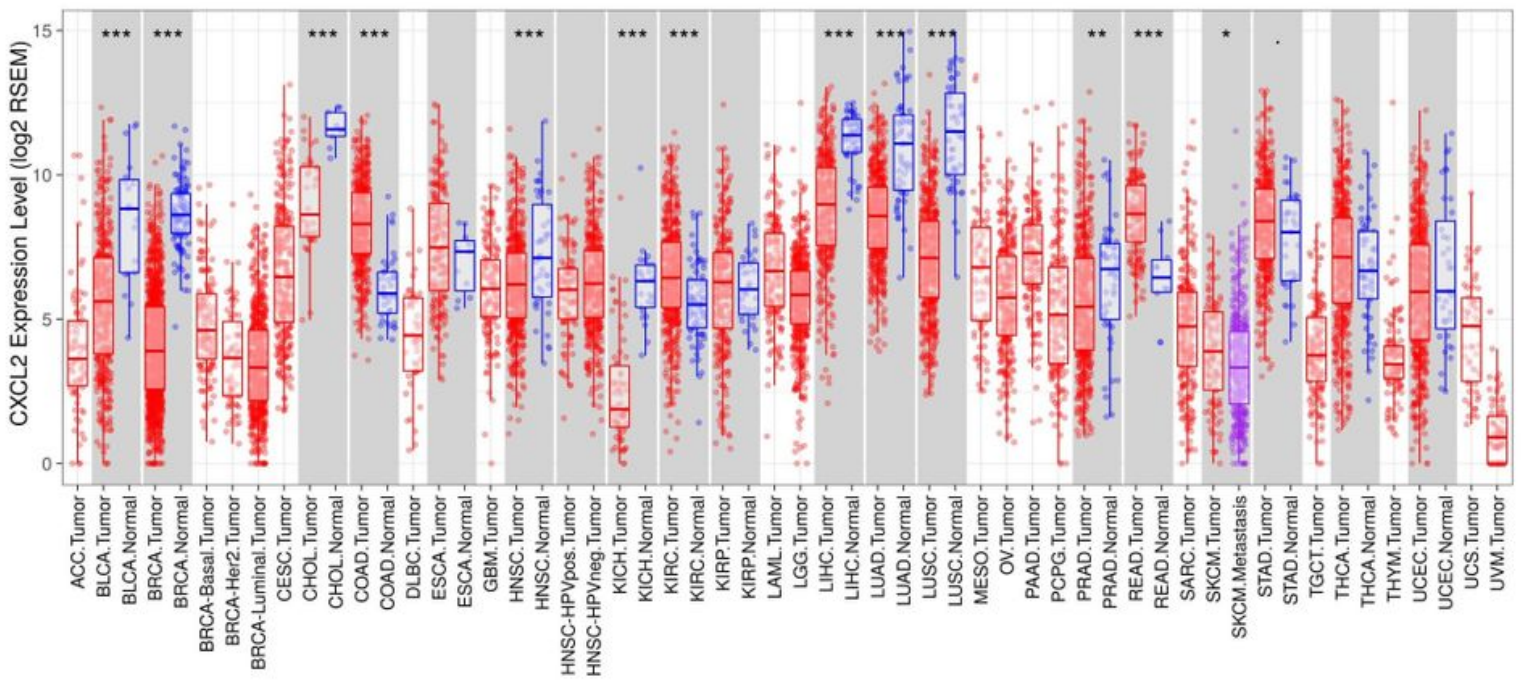

Figure 1

CXCL2 expression levels in different cancers. (A) CXCL2 expression in data sets of different cancers compared with normal tissues in the Oncomine database. (B) CXCL2 expression levels in different tumor types from TCGA database were determined by TIMER $\left({ }^{\star} P<0.05,{ }^{* *} P<0.01,{ }^{*} * P^{2}<0.001\right)$.
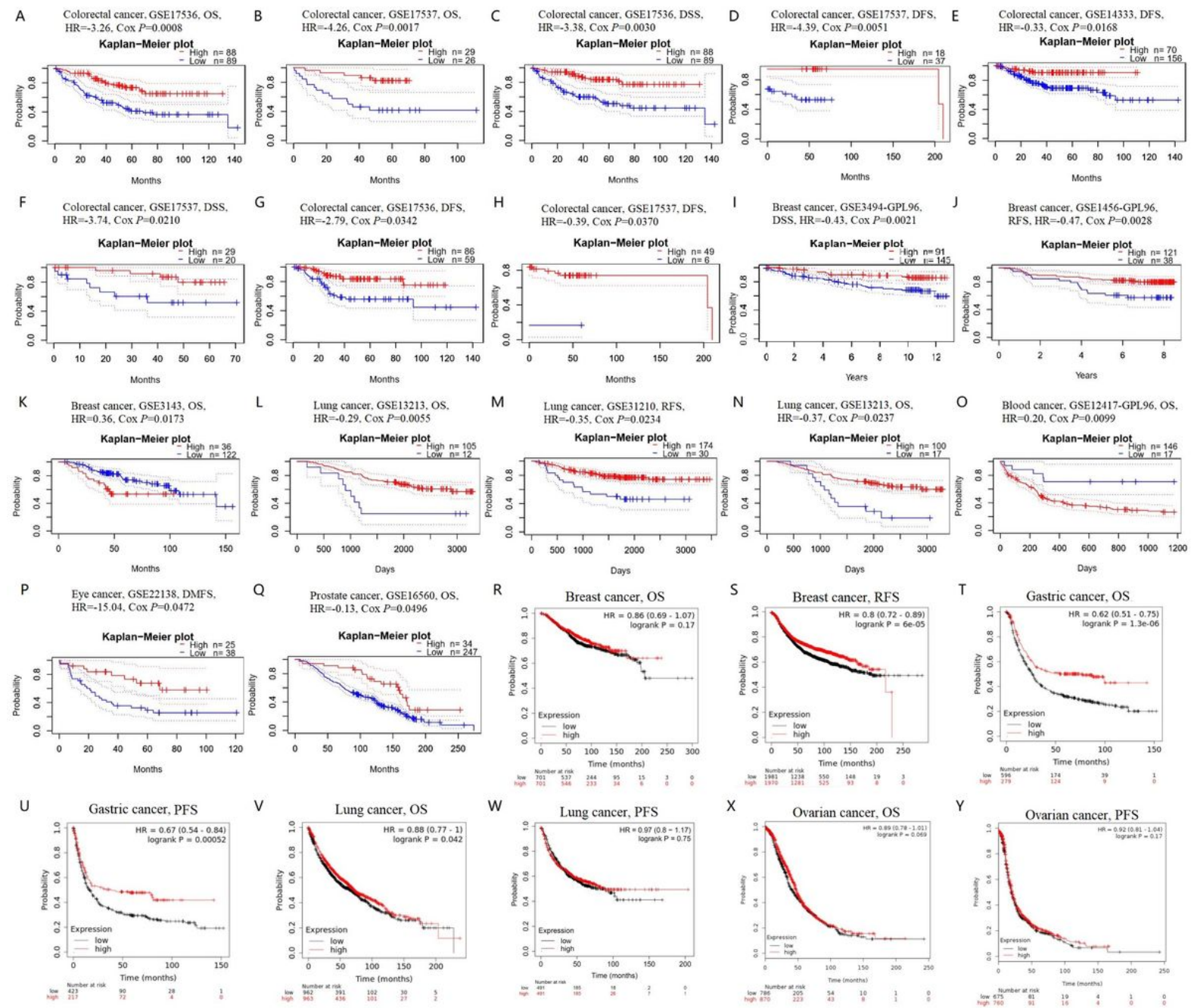

Figure 2 
Kaplan-Meier survival curves comparing the expression of CXCL2 in different cancers in the PrognoScan (A-Q) and Kaplan-Meier plotter databases (R-Y). (A-H) Survival curves of OS, DSS, and DFS in three colorectal cancer datasets (GSE17536, GSE17537 and GSE14333). (I-K) Survival curves of DSS, RFS and OS in three breast cancer datasets (GSE3494-GPL96, GSE1456-GPL96 and GSE3143). (L-N) Survival curves of RFS and OS in two lung cancer datasets (GSE13213 and GSE31210). (O-Q) High CXCL2 expression was correlated with poor OS in blood cancer cohort (GSE12417-GPL96), better DMFS in eye cancer cohort (GSE22138) and better OS in prostate cancer cohort (GSE16560). (R,S) OS and RFS survival curves of breast cancer. (T,U) OS and PFS survival curves of gastric cancer. $(\mathrm{V}, \mathrm{W})$ OS and PFS survival curves of lung cancer. $(\mathrm{X}, \mathrm{Y})$ OS and PFS survival curves of ovarian cancer.

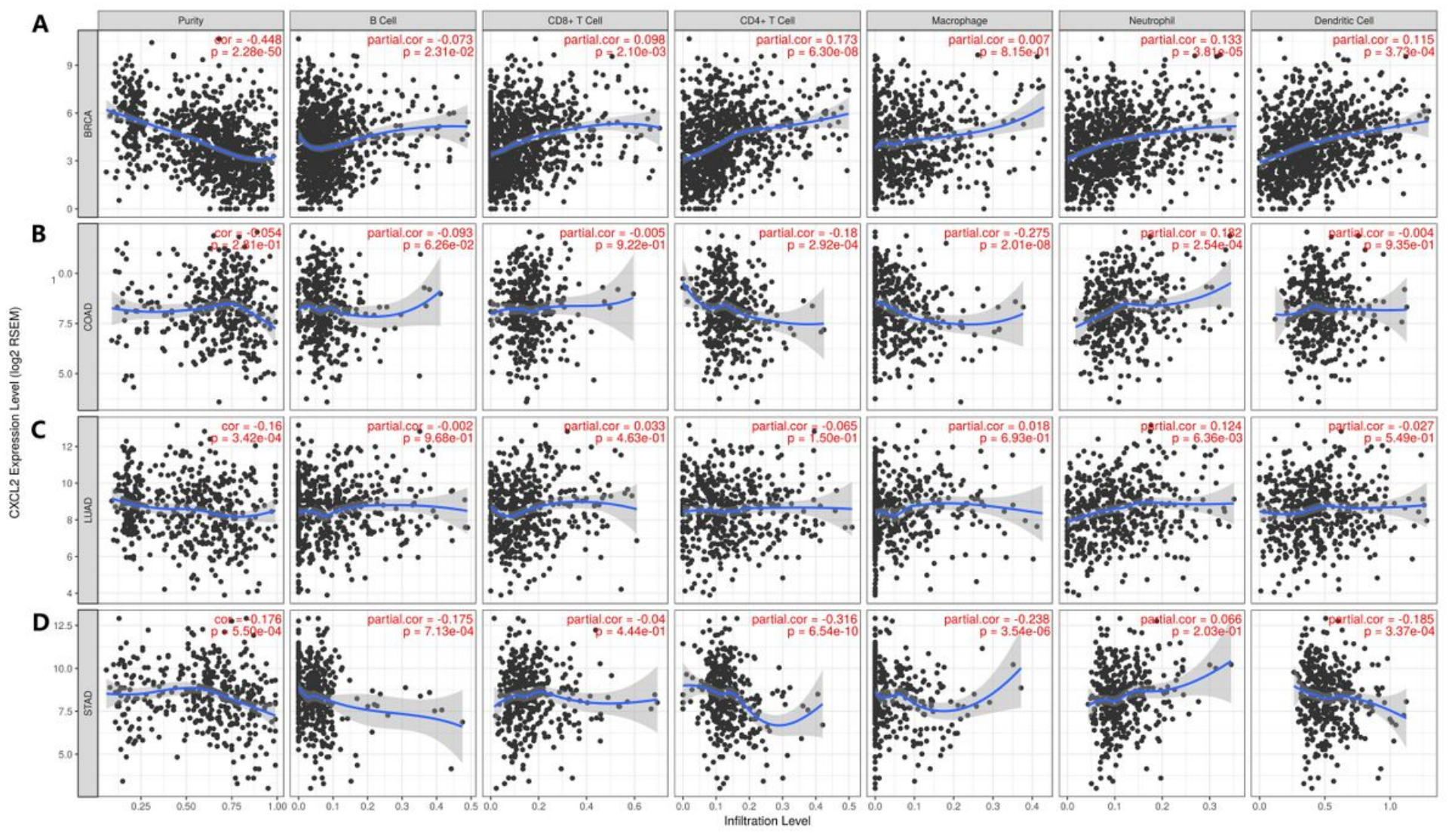

Figure 3

Correlation of CXCL2 expression with immune infiltration level in BRCA, COAD, LUAD, and STAD. (A) CXCL2 expression was positively correlated with the infiltration of $B$ cells, CD8+T cells, CD4+T cells, neutrophils and DCs in BRCA. (B) CXCL2 expression has no significant correlations with tumor purity and infiltrating levels of B cells, CD8+T cells, and DCs. It showed correlations with CD4+T cells, macrophages, neutrophils level in COAD. (C) CXCL2 expression has no significant correlations with infiltrating levels of B cells, CD8+T cells, CD4+T cells, macrophages and DCs, but only a weak correlation with neutrophils in LUAD. (D) CXCL2 expression has negative correlations with infiltration of B cells, CD4+ T cells, macrophages, and DCs in STAD. 


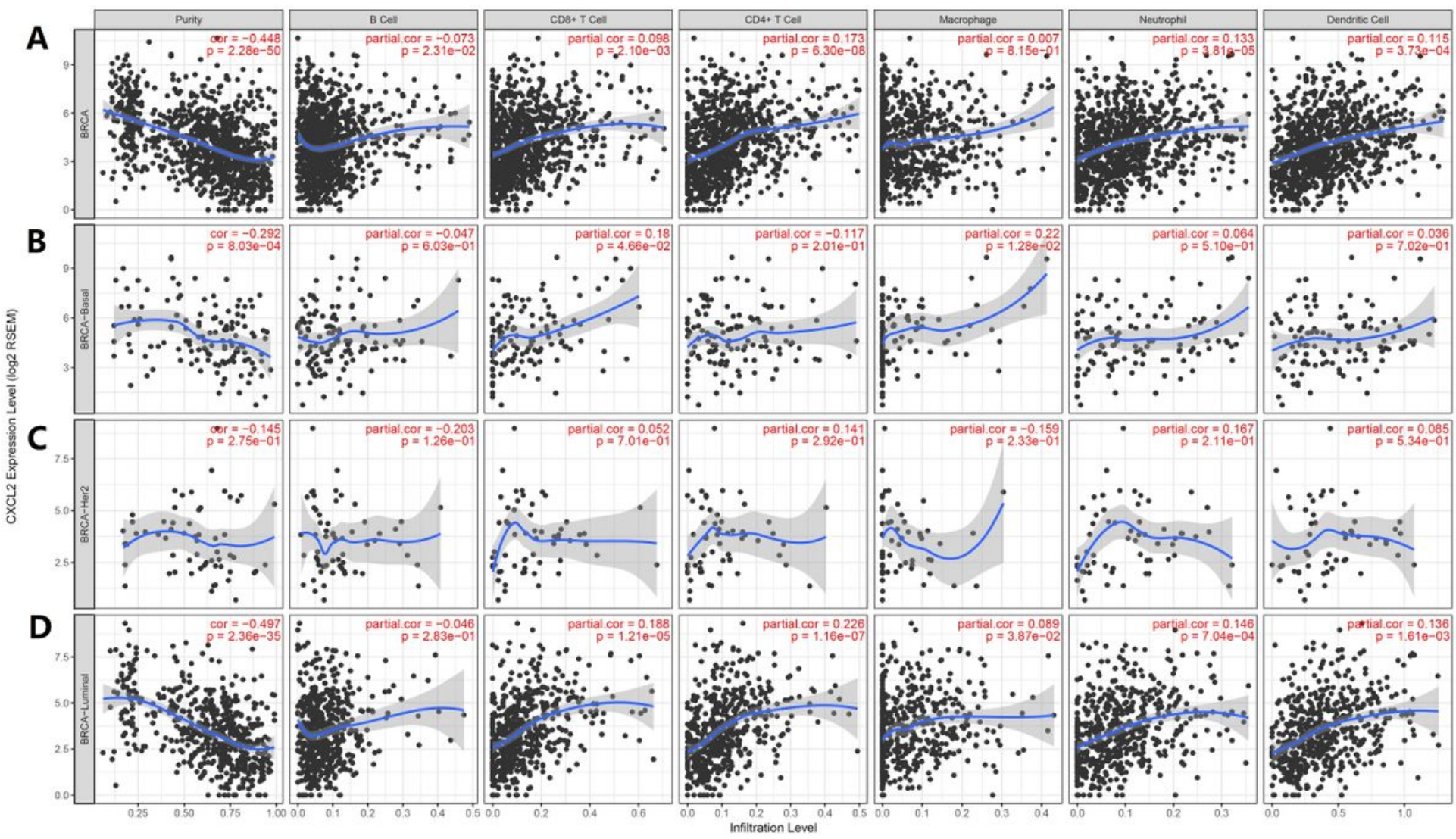

Figure 4

Correlation between the expression of CXCL2 and the immune infiltration of different types of BRCA. (A) CXCL2 expression was correlated with the infiltration of $B$ cells, CD8+T cells, CD4+T cells, neutrophils and DCs in BRCA. (B) CXCL2 expression has no significant correlations with infiltrating levels of B cells, CD4+T cells, neutrophils and DCs, but only a very weak correlation with CD8+T cells and macrophages in BRCA-Basal. (C) CXCL2 expression has no significant correlations with immune infiltrating level in BRCA-Her2. (D) CXCL2 expression was positively correlated with the infiltration of CD8+T cells, CD4+T cells, macrophages, neutrophils and DCS in BRCA-Luminal.

展

Figure 5

CXCL2 expression correlated with macrophage polarization in BRCA, COAD, LUAD and STAD. Markers include CD86 and CSF1R of monocytes; CCL2, CD68, and IL10 of TAMs; NOS2, IRF5, and PTGS2 of M1 macrophages; and CD163, VSIG4, and MS4A4A of M2 macrophages. (A) Scatterplots of correlations between CXCL2 expression and gene markers of M2 in the above four cancers. (B) Scatterplots of correlations between CXCL2 expression and gene markers of M2 in the above four cancers. (C) Scatterplots of correlations between CXCL2 expression and gene markers of TAMs in the above four cancers. (D) Scatterplots of correlations between CXCL2 expression and gene markers of Monocytes in the above four cancers.

A

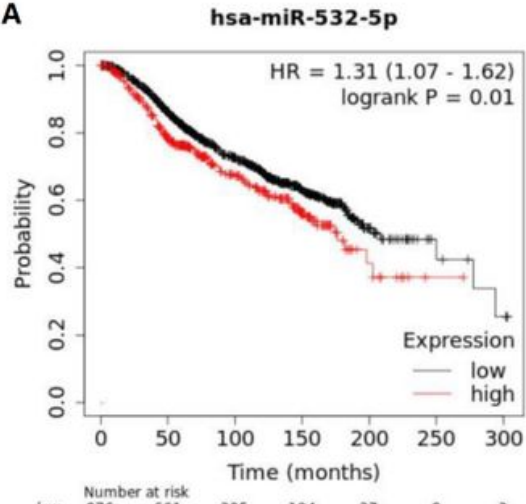

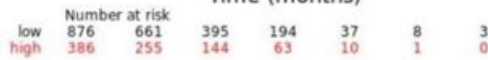
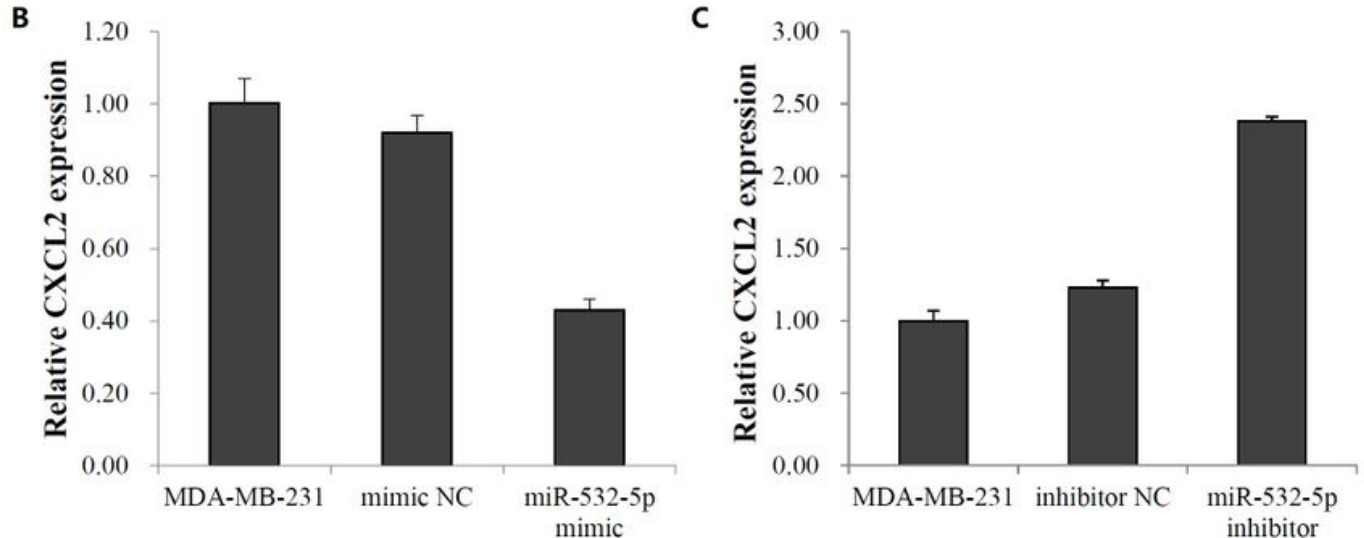

Figure 6 
The role of miR-532-5p on prognosis and its regulation on CXCL2. (A) Effect of miR-532-5p on overall survival rate of patients. It has negative correlation with OS in BRCA. $(B, C)$ The level of CXCL2 mRNA decreased after overexpression of miR-532-5p, and after inhibition of miR-532-5p expression, the level of CXCL2 mRNA increased 